\title{
Prediction of hidden multiferroic order in graphene zigzag ribbons
}

\author{
J. Fernández-Rossier \\ Departamento de Física Aplicada, Universidad de Alicante, 03690 Alicante, Spain
}

(Received 7 December 2007; published 27 February 2008)

\begin{abstract}
An electronic phase with coexisting magnetic and ferroelectric order is predicted for graphene ribbons with zigzag edges. The electronic structure of the system is described with a mean-field Hubbard model that yields results very similar to those of density functional calculations. Without further approximations, the mean-field theory is recasted in terms of a BCS wave function for electron-hole pairs in the edge bands. The BCS coherence present in each spin channel is related to spin-resolved electric polarization. Although the total electric polarization vanishes, due to an internal phase locking of the BCS state, strong magnetoelectric effects are expected in this system. The formulation naturally accounts for the two gaps in the quasiparticle spectrun, $\Delta^{0}$ and $\Delta^{1}$, and relates them to the intraband and interband self-energies.
\end{abstract}

DOI: 10.1103/PhysRevB.77.075430

PACS number(s): 71.30.+h, 75.50.Gg, 75.75.+a

\section{INTRODUCTION}

The quest of novel electronic phases, characterized by new order parameters, and the interplay between electric and magnetic degrees of freedom are two of the major themes in condensed matter physics. The coexistence of magnetic and electric orders is associated with transition metal perovskites ${ }^{1}$ whereas spintronics proposals are based on materials where either spin-orbit interactions ${ }^{2-4}$ or $d$ electrons ${ }^{5}$ play a prominent role. Here, I show that the magnetic ground state predicted ${ }^{6-12}$ in graphene zigzag ribbons, a chemically simple system without $d$ electrons and negligible spin-orbit coupling, is indeed an electronic phase whose order parameter is the product of the spin and the electric polarization. This electronic state may be studied experimentally, thanks to recent progress in the fabrication of graphene ${ }^{13-15}$ and graphene based flat nanostructures. ${ }^{15-17}$

Early theoretical work predicts that graphene is a zero gap semiconductor, with electron-hole symmetry and linear conduction and valence bands. These features arise naturally from a tight-binding model with one $\Pi_{z}$ orbital per atom in a honeycomb lattice at half filling, and they are related to the fact that the honeycomb lattice is bipartite. The electronic structure of graphene nanoribbons depends dramatically on their atomic strucuture ${ }^{18-21}$ Here, I focus on graphene ribbons with zigzag edges. The single-particle description of this system features two almost degenerate quasiflat bands at the Fermi energy. ${ }^{18-20}$ These flat bands are associated with edge states. When Coulomb repulsion is added to this picture within a mean-field Hubbard model, ${ }^{6-8}$ local moments of oppposite signs form in the edges, with a total zero spin, and a gap opens at the Fermi energy. The predictions of this model are robust with respect to the addition of more orbitals, second-neighbor hoppings, and long-range Coulomb interactions, all present in density functional (DFT) calculations. $^{9-12}$ DFT results and the mean-field Hubbard model yield very similar results for the low energy sector of the electronic structure both for zigzag graphene ribbons ${ }^{22}$ and nanoislands. ${ }^{23}$ This permits a significant computational simplification as well as conceptual advantage through the use of exact results valid for the Hubbard model. ${ }^{24}$

In this paper, three things are done. In the first place, I show that the mean-field wave function of the Hubbard model for graphene ribbons with zigzag edges is that of two phase locked BCS condensates of spin-polarized electronhole pairs living in the edge bands, the only bands affected by the interactions. Second, the BCS electron-hole coherence implicit in the wave funcion is associated with the existence of spin-resolved transverse electric polarizations that yield a zero total electric dipole and spin when summed. Therefore, the standard mean-field magnetic phase of zigzag graphene ribbons $^{6-12}$ is an excitonic insulator phase with a hidden ferroelectric order. Third, the mean-field bands are written in terms of the BCS gap and diagonal self-energies.

\section{ELECTRONIC STRUCTURE OF ZIGZAG RIBBONS}

Zigzag graphene ribbons are described with a singleorbital tight-binding model ${ }^{18,20}$ plus an on-site Hubbard repulsion treated in the mean-field approximation at half filling, $6,7,23$

$$
\mathcal{H}=\sum_{\vec{r}, \vec{r}^{\prime}, \sigma} t_{\vec{r}, \vec{r}^{\prime}} c_{\vec{r} \sigma^{\prime}}^{\dagger} c_{\vec{r}^{\prime} \sigma}+U \sum_{\vec{r}} n_{\vec{r}, \uparrow}\left\langle n_{\vec{r}, \downarrow}\right\rangle+n_{\vec{r}, \downarrow}\left\langle n_{\vec{r}, \uparrow}\right\rangle,
$$

where $c_{\vec{r} \sigma}^{\dagger}$ creates an electron at the $\Pi_{z}$ orbital of atom located at $\vec{r}$ with spin $\sigma$ and $n_{\vec{r}, \sigma}=c_{\vec{r} \sigma}^{\dagger} c_{\vec{r} \sigma}$ is the occupation operator. The first term in the Hamiltonian describes the firstneighbor hopping $(t=2.5 \mathrm{eV})$ in the graphene ribbon and the second describes the on-site Coulomb repulsion. This model describes hydrogen passivated graphene ribbons. A value of $U=2 \mathrm{eV}$ yields results in agreement with DFT., ${ }^{9}, 10$ Qualitatively, the main results of this work do not change as long as $U<2.2 t$, above which two dimensional graphene becomes antiferromagnetic. The zigzag ribbon is a one dimensional crystal whose unit cell, shown in Fig. 1(a), is repeated along the $x$ direction. The position $\vec{r}$ is determined by a unit cell index $x$ and an intracell index $I$. Notice that the top and bottom atoms belong to different sublattices. In a unit cell, there are $N_{P}$ pairs of $A$ and $B$ atoms, and the width of the ribbon is $W \simeq \sqrt{3}(N) a$, with $N=2 N_{P}$.

\section{A. Numerical results}

The spectrum of the self-consistent mean-field Hamiltonian for a ribbon with $N$ atoms per unit cell has $N$ bands 

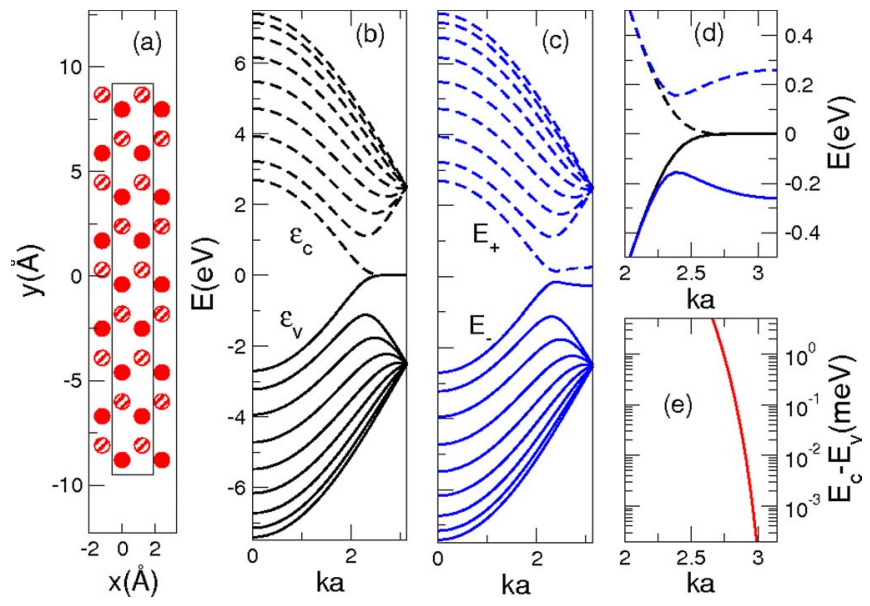

FIG. 1. (Color online) (a) Zigzag ribbon unit cell. (b) Bands of $N=18$ zigzag ribbons with $U=0$ (c) and $U=2 \mathrm{eV}$. Only half of the Brillouin zone is shown. (d) Comparison of low energy bands. Interacting bands have been shift downward by $U / 2$. (e) $U=0$ singleparticle gap at the edge states.

per spin channel, half of which are occupied. Figure 1(b) shows the well-known noninteracting $(U=0)$ bands for a ribbon with $N=18$. Solid (dashed) lines represent full (empty) states. The two flat bands in the outer region of the Brillouin zone correspond to states that are localized in the edges of the ribbon. ${ }^{18}$ As shown in Fig. 1(d), they are not really degenerate except for $k a= \pm \pi$. At zero temperature, the lower (valence) band is full and the upper (conduction) band is empty. The operators that annihilate an electron in those bands are

$$
\begin{gathered}
e_{k, \sigma}=C_{k \sigma} \equiv \frac{1}{\sqrt{L}} \sum_{x, I} e^{i k x} \psi_{c k}(I) c_{x, I, \sigma}, \\
h_{k, \sigma}^{\dagger}=V_{k \sigma} \equiv \frac{1}{\sqrt{L}} \sum_{x, I} e^{i k x} \psi_{v k}(I) c_{x, I, \sigma},
\end{gathered}
$$

where $\frac{1}{\sqrt{L}} e^{i k x} \psi_{(c, v), k}(I)$ are the Bloch eigenstates and $L$ is the length of the ribbon. The gap between these bands is proportional to the penetration of the edge states toward the bulk region. ${ }^{18}$

Figure 1(c) shows the mean-field interacting bands, shifted rigidly by $-U / 2$. They are obtained by numerical solution of the self-consistent mean-field Hamiltonian. A gap opens at the Fermi energy, in agreement with DFT calculations. $^{9-12}$ The average spin-resolved charges along a unit cell are shown in Figs. 2(a) and 2(c). Spin up (down) electrons pile at the top (bottom) edge of the ribbon and leave a charge deficit in the opposite side, also in agreement with DFT calculations. Therefore, the edges have local magnetization with opposite sign. For a given spin, there is an excess of electrons in one edge, which are missing in the other. The total electronic charge turns out to be the same in all the atoms.
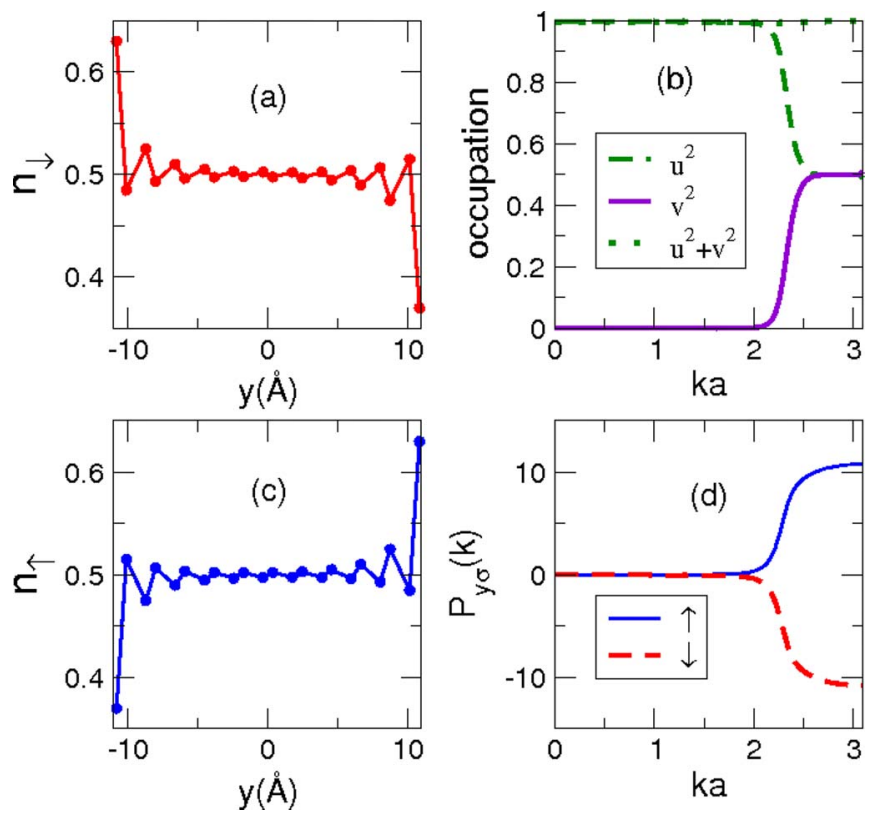

FIG. 2. (Color online) [(a) and (c)] Spin revolved occupation $n_{I \sigma}$ as a function of vertical position in unit cell for $N=22$ ribbons. (b) Occupation factors $v_{\sigma}(k)^{2}, u_{\sigma}(k)^{2}$, and $v_{\sigma}(k)^{2}+u_{\sigma}(k)^{2}$. (d) Spinresolved dipole $\mathcal{P}_{y \sigma}(k)$.

\section{B. Derivation of the BCS state}

It is crucial to realize that the noninteracting and the shifted mean-field bands are identical except for the lowest energy empty band and the highest energy occupied band which differ in the outer sector of the Brillouin zone, as shown in Fig. 1(c). These bands are denoted by - and + and $v$ and $c$ for the interacting and the noninteracting cases. It turns out that both - and + can be expressed as linear combinations of $c$ and $v$ only, with an accuracy better than $99 \%$. Thus, it is possible to relate the two interacting states, - and + , with the noninteracting conduction and valence bands through

$$
\left(\begin{array}{c}
f_{-, k \sigma}^{\dagger} \\
f_{+, k \sigma}^{\dagger}
\end{array}\right)=\left(\begin{array}{cc}
u_{k} & v_{k} e^{i \phi_{\sigma}} \\
-v_{k}^{*} e^{-i \phi_{\sigma}} & u_{k}^{*}
\end{array}\right)\left(\begin{array}{c}
V_{k \sigma}^{\dagger} \\
C_{k \sigma}^{\dagger}
\end{array}\right) .
$$

Importantly, the spin dependence is limited to the phases. The moduli of the coefficients, $\left|u_{k}\right|^{2}$ and $\left|v_{k}\right|^{2}$, are shown in Fig. 2(b). I find that $v_{k}^{2}+u_{k}^{2}>0.99$. Using this relation, I formulate the interacting theory in terms of electrons and holes in the noninteracting valence and conduction bands. The mean-field ground state, which is formed by filling all the mean-field bands below the gap, is written as $|\Phi\rangle=|\Phi\rangle_{\downarrow} \times|\Phi\rangle_{\uparrow}$, where

$$
|\Phi\rangle_{\sigma}=\prod_{k, \nu=1, N / 2} f_{\nu, k, \sigma}^{\dagger}|0\rangle=\prod_{k} f_{-, k, \sigma}^{\dagger}\left|G^{\prime}\right\rangle,
$$

where $\left|G^{\prime}\right\rangle=\prod_{k, \nu=1, N / 2-1} f_{\nu, k, \sigma}^{\dagger}|0\rangle$ denotes the state where all the bands below $V$ (or - ) in Figs. 1(b) and 1(c) are full. Making use of Eq. (3), I write 


$$
|\Phi\rangle=\prod_{k, \sigma}\left(u_{k} V_{k, \sigma}^{\dagger}+v_{k} e^{i \phi_{\sigma}} C_{k, \sigma}^{\dagger}\right)\left|G^{\prime}\right\rangle .
$$

The next step is to factor out the $V^{\dagger}$ operator,

$$
|\Phi\rangle=\prod_{k, \sigma}\left(u_{k}+v_{k} e^{i \phi_{\sigma}} C_{k, \sigma}^{\dagger} V_{k, \sigma}\right) V_{k, \sigma}^{\dagger}\left|G^{\prime}\right\rangle .
$$

I now identify inside Eq. (6) the noninteracting ground state $|G\rangle_{0}=\prod_{k, \sigma} V_{k, \sigma}^{\dagger}\left|G^{\prime}\right\rangle$ with no holes in the noninteracting valence band and no electrons in the noninteracting conduction band. I thus can relate the interacting mean-field ground state to the noninteracting ground state,

$$
|\Phi\rangle=\prod_{k, \sigma}\left(u_{k}+v_{k} e^{\left.i \phi_{\sigma} C_{k, \sigma}^{\dagger} V_{k, \sigma}\right)|G\rangle_{0} .}\right.
$$

As a final step, I use the electron-hole notation defined in Eq. (2) instead of the conduction and valence band notations. I obtain

$$
|\Phi\rangle=\prod_{k, \sigma}\left(u_{k}+v_{k} e^{i \phi_{\sigma}} e_{k, \sigma}^{\dagger} h_{k, \sigma}^{\dagger}\right)|G\rangle_{0} .
$$

Equation (8) is one of the important results of this work; the mean-field ground state implicit in Eq. (1) that yields the bands of Fig. 1(c) and the spin-density profiles of Figs. 2(a) and 2(c) can be written as the product of two BCS condensates of spin-polarized electron-hole pairs in the two noninteracting bands closest to the Dirac point. Notice that, a priori, the low energy mean-field states obtained by numerical diagonalization of the mean-field Hamiltonian could be a linear combination of all the noninteracting states, not only the two low energy bands. This decoupling is not an approximation of this work but an outcome of the numerical calculation.

\section{PHYSICAL CONSEQUENCES}

The state [Eq. (8)] is found in the context of excitonic insulators $^{25}$ and nonequilibrium exciton condensates. ${ }^{26}$ It portrays the transition from Figs. 1(b) and 1(c) as an excitonic insulator instability. In real space, electrons with spin $\sigma$ and holes with $\bar{\sigma}$ are localized in one of the edges of the ribbon; the electron-hole pairs with opposite spins are localized in the other. Importantly, this BCS state implies the existence of nonmagnetic long-range order for the interband operators. The numerical calculations systematically show that the interband coherence,

$$
\left\langle\Phi\left|e_{k \uparrow}^{\dagger} h_{k \uparrow}^{\dagger}\right| \Phi\right\rangle=v_{k}^{*} u_{k} e^{-i \phi_{\uparrow}}=-\left\langle\Phi\left|e_{k \downarrow}^{\dagger} h_{k \downarrow}^{\dagger}\right| \Phi\right\rangle,
$$

is finite and their relative phase is locked: $\left(\phi_{\uparrow}-\phi_{\downarrow}\right)=\pi$. Interband coherence is zero for $U=0$ and is related to observables that mix the valence and conduction bands. These acquire an anomalous expectation value in the $U>0$ phase.

\section{A. Spin-dipole operator}

The interband coherence is associated with electric polarization ${ }^{27}$ in nonequilibrium exciton condensates and to electronic ferroelectricity in the case of the Bose condensation of slave bosons in the case of mixed-valence compounds. ${ }^{28}$ Hence, I look for the connection between the interband coherence implicit in Eq. (8) and the spin-resolved electric dipole implicit in Figs. 2(a) and 2(c). The electric dipole is written as the sum of the spin-resolved dipole $\mathcal{P}_{y}=\mathcal{P}_{y \uparrow}+\mathcal{P}_{y \downarrow}$, where

$$
\mathcal{P}_{y \sigma}=\sum_{x, I} y_{I} e n_{x, I, \sigma}=\sum_{k, \nu, \nu^{\prime}} d_{\nu, \nu^{\prime}} C_{k, \nu, \sigma}^{\dagger} C_{k, \nu^{\prime}, \sigma}
$$

are the spin-resolved components of the dipole operator, and the dipole matrix elements are given by

$$
d_{\nu, \nu^{\prime}}(k)=\sum_{I} e y_{I} \psi_{k, \nu}^{*}(I) \psi_{k, \nu^{\prime}}(I) .
$$

The labels $\nu, \nu^{\prime}$ run over the noninteracting bands. The ribbon is centered at $y=0$. Because of the mirror symmetry of the zigzag unit cell, $d_{\nu \nu}(k)=0$, so that only the band-mixing terms on Eq. (11) can yield a contribution. The average dipole operator in the state [Eq. (8)] is

$$
\left\langle\mathcal{P}_{y}\right\rangle=\frac{1}{L} \sum_{k, \sigma} d_{C V}(k) u_{k} v_{k}^{*} e^{-i \phi_{\sigma}}+\text { H.c. }=\frac{1}{L} \sum_{k, \sigma} \mathcal{P}_{y \sigma}(k),
$$

whereas $\left\langle\mathcal{P}_{y}\right\rangle=0$ the spin resolved components $\mathcal{P}_{y \sigma}(k)$, shown in Fig. 2(d), are finite and with opposite sign. A zero net dipole resulting for the sum of two opposite spinresolved dipoles is expected from inspection of Figs. 2(a) and 2(c) and the homogeneous spin-summed charge distribution. Thus, the spin-resolved dipoles are related to the interband coherence, and the absence of the net electric dipole is related to their phase locking in Eq. (9). In order to characterize this kind of electronic order, I introduce the spindipole operator,

$$
\mathcal{P}_{\sigma, \sigma^{\prime}}(y, z)=e \sum_{I} y_{I} n_{I \sigma} S_{\sigma, \sigma^{\prime}}^{z}=\left(\begin{array}{cc}
\mathcal{P}_{y \uparrow} & 0 \\
0 & -\mathcal{P}_{y \downarrow}
\end{array}\right),
$$

where $S_{\eta, \eta^{\prime}}^{z}$ is the Pauli matrix. Thus, the relevant order parameter associated with the electronic state [Eq. (8)] is $\operatorname{Tr}_{\sigma}\langle\Phi|\mathcal{P}(y, z)| \Phi\rangle$. Spin rotational invariance permits one to choose $z$ along any direction in the spin space. This order parameter is invariant under the combined action of time reversal and mirror symmetry and provides a natural explanation to the spin polarization of the system when subject to a transverse electric field, predicted by DFT calculations. Notice that this phase is different from the nonmagnetic ferroelectric phase predicted in Eq. (8), which is not found in DFT.

\section{B. Origin of the gaps}

The mean-field state [Eq. (8)] invites one to write the interacting bands in terms of a BCS-like gap related to interband coherence. To do that, I project out all the bands except $C$ and $V$,

$$
c_{x I, \sigma}^{\dagger} \simeq \frac{1}{\sqrt{L}} \sum_{k} e^{-i k x}\left(\psi_{k, C}(I) C_{k \sigma}^{\dagger}+\psi_{k, V}(I) V_{k \sigma}^{\dagger}\right) .
$$

The occupation of the sites is expressed as $n_{I \sigma}=\frac{1}{2}+\sigma m_{I}$, where $\sigma= \pm$. This automatically ensures that the occupation 
in each site is 1. Using transformation [Eq. (14)], the meanfield Hamiltonian reads

$$
\mathcal{H}=\sum_{k, \sigma}\left(C_{k, \sigma}^{\dagger}, V_{k, \sigma}^{\dagger}\right)\left(\begin{array}{cc}
\xi_{c \sigma}(k) & \Delta_{\sigma}(k) \\
\Delta_{\sigma}^{*}(k) & \xi_{v \sigma}(k)
\end{array}\right)\left(\begin{array}{c}
C_{k, \sigma} \\
V_{k, \sigma}
\end{array}\right),
$$

with

$$
\xi_{\nu \sigma}(k)=\epsilon_{\nu}(k)+\frac{U}{2}+U \bar{\sigma} \sum_{I}\left|\psi_{k, \nu}(I)\right|^{2}\left\langle m_{I}\right\rangle,
$$

where $\epsilon_{\nu}(k)$ are the $U=0$ bands and $\nu=c, v$. The second term in Eq. (16) is the rigid shift of the bands $U_{2}^{n}$ and the third term is the diagonal self-energy $\Sigma_{\nu, \sigma}(k)$. The off-diagonal self-energy reads

$$
\Delta_{\sigma}(k)=\bar{\sigma} U \sum_{I} \psi_{k, c}(I) \psi_{k, v}^{*}(I)\left\langle m_{I}\right\rangle .
$$

Notice that $\Delta_{\sigma}(k)=-\Delta_{\bar{\sigma}}(k)$, which explains the phase locking of Eq. (9). Notice also that in the Hubbard model, the selfenergies for spin $\sigma$ electrons depend on the density of carriers with opposite spin $\bar{\sigma}$. For each $k \sigma$, the mean-field two by two matrix can be written as $\frac{U}{2}+\vec{h}_{\sigma}(k) \vec{\tau}$, where $\tau$ are the Pauli matrices, and the effective field can be written as

$$
\vec{h}_{\sigma}(k)=\left(\operatorname{Re}\left[\Delta_{\sigma}(k)\right], \operatorname{Im}\left[\Delta_{\sigma}(k)\right], \frac{\xi_{c, \sigma}-\xi_{v, \sigma}(k)}{2}\right) .
$$

The eigenvalues of this two by two matrix are

$$
E_{ \pm, \sigma}(k)=\frac{1}{2}\left(U \pm \sqrt{\left[\xi_{c, \sigma}-\xi_{v, \sigma}(k)\right]^{2}+4\left|\Delta_{\sigma}(k)\right|^{2}}\right) .
$$

The transformation [Eq. (3)] permits one to diagonalize [Eq. (15)], obtaining $\mathcal{H}=\Sigma_{k, \sigma, \tau= \pm} E_{\tau, \sigma}(k) f_{k \tau \sigma}^{\dagger} f_{k \tau \sigma}$. At zero temperature, only the lower branches $E_{-, \sigma}(k)$ are occupied. The mean-field dispersion $E_{\tau \sigma}(k)$ depends on $\xi_{\nu \sigma}(k)$ and on $\Delta_{\sigma}(k)$ which, in turn, depend on the magnetization,

$$
m(I)=\sum_{k} \psi_{k, c}^{*}(I) \psi_{k, v}(I) u_{k} v_{k}^{*}+\text { H.c. }
$$

The magnetization depends on the transformation factors, $u$ and $v$, which depend on the energies through

$$
\left|v_{k}\right|^{2}=\frac{1}{2}\left(1-\frac{h_{z \sigma}(k)}{\left|\vec{h}_{\sigma}(k)\right|}\right) u_{k} v_{k} e^{i \phi_{\sigma}}=\frac{-h_{x, \sigma}(k)}{\left|\vec{h}_{\sigma}(k)\right|} .
$$

Equations (16)-(20) form a self-consistent set. The numerical solutions of the mean-field Hubbard model [Eq. (1)] also satisfy these equations. This permits one to relate the mean-field dispersion to the diagonal self-energy $\left|\Sigma_{c, \sigma}(k)-\Sigma_{v, \sigma}(k)\right|$ and the off-diagonal self-energy $|\Delta(k)|$, as shown in Fig. 3. They are spin independent. It is apparent that these self-energies are finite in different regions of the Brilloiun zone. The diagonal self-energy is related to the nonhybridized edge states located in the outer region of the Brillouin zone, whereas the off-diagonal self-energy occurs for weakly hybridized edge states, at smaller $|k|$. Further reduction of $|k|$ opens the single-particle gap, which overshades the self-energies. These results also permit one to
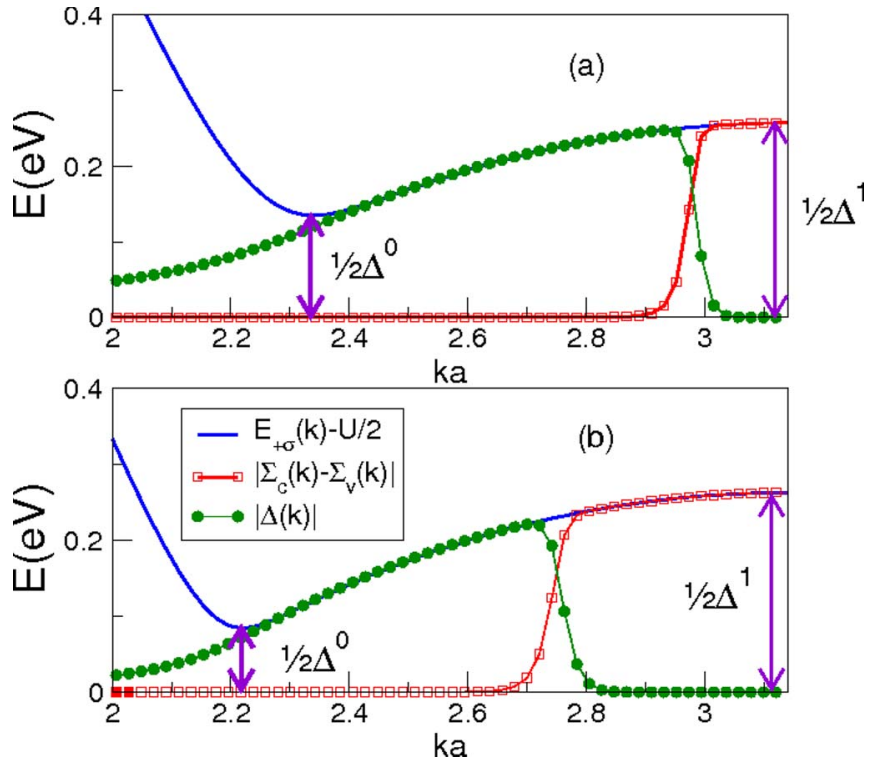

FIG. 3. (Color online) Relation between dispersion $E_{+\sigma}(k)$ and the diagonal $\Sigma_{\nu \sigma}(k)$ and off-diagonal $\Delta(k)$ self-energies for two ribbons with (a) $N=22$ and (b) $N=42$.

unveil the origin of the gaps $\Delta^{1}$ and $\Delta^{0}$ introduced in Eq. (10) (see Fig. 3). It is apparent that the $\Delta^{1}$ gap is given by the diagonal self-energy whereas the $\Delta^{0}$ gap is related to the nondiagonal self-energy. Accordingly, $\Delta^{1}$ is insensitive to the ribbon width and can be approximated by $\Delta^{1} \simeq 2 U|m|$, where $|m|$ is the magnetization of the edge atoms. In contrast, $\Delta^{0}$ decreases as the ribbon width increases due to the smaller interedge hybridization.

\section{DISCUSSION AND CONCLUSIONS}

The long-range order implicit in this and previous meanfield theories of graphene zigzag ribbons ${ }^{6,7,9-12}$ is known to be destroyed in one dimension because of long-wavelength spin wave modes ${ }^{7}$ associated with the breaking of a continous symmetry. However, the results of these calculations are still relevant, since they hold true in finite length ribbons and open-end nanotubes, as opposed to infinite length systems considered here. ${ }^{30}$ In particular, I have verified that the existence of a spin-resolved dipole, and the decoupling of the edge states from the rest, which permits one to write the mean-field ground state as an excitonic insulator state, remain valid for finite length graphene ribbons and open-end nanotubes, and in short zigzag ribbons attached to infinite nonmagnetic graphene, similar to armchair ribbon heterostructures. ${ }^{33}$ The presence of local magnetic order in short zigzag graphene edges has been confirmed now in a variety of DFT calculations. ${ }^{23,29,31,32}$ The lack of long-range order is not an issue in systems whose size is smaller than the spin correlation length, which is in the range of $10 \mathrm{~nm}$ at $100 \mathrm{~K}$, according to recent DFT calculations. ${ }^{32} \mathrm{I}$ have also verified that the introduction of a second-neighbor hopping ${ }^{34}$ $t^{\prime}$ yields energy bands in better agreement with DFT results without changing the physical picture described in this work: the mean-field ground state can still be written as a BCS state 
[Eq. (8)] which is related to the finite spin-dipole operator, whose expectation value is quite insensitive to the value of $t^{\prime}$ in the range $t^{\prime} \ll t$.

To summarize, the ground state of graphene zigzag ribbons features a kind of electronic order, with local magnetic moments and spin-resolved electric polarization, characterized by an order parameter, the spin dipole [Eq. (13)]. This finding arises from the mapping of the widespread ${ }^{6-8}$ meanfield Hubbard model wave function, which yields results very similar to DFT calculations, ${ }^{9-12}$ into a BCS state of two spin-polarized electron-hole condensates. The BCS coherence is intimately related to the spin-resolved ferroelectricity, and the relative phase locking of the two spin channels accounts for cancellation of the total dipole. The electronic order naturally accounts for the electric-induced spin polar- ization predicted by DFT calculations. The joint presence of electric and magnetic orders, normally associated with chemically complex multiferroic materials, permits one to anticipate other strong magnetoelectric effects in graphene ribbons.

\section{ACKNOWLEDGMENTS}

Fruitful conversations with J. J. Palacios and L. Brey are acknowleged. This work has been financially supported by MEC-Spain (Grant No. MAT2007-65487 and Ramon y Cajal Program), by Generalitat Valenciana (No. Accomp07-054), by Consolider (No. CSD2007-0010), and, in part, by FEDER funds.
${ }^{1}$ S. Cheong and M. Mostovoy, Nat. Mater. 6, 13 (2007).

${ }^{2}$ S. Datta and B. Das, Appl. Phys. Lett. 56, 665 (1990).

${ }^{3}$ B. A. Bernevig, T. L. Hughes, and S. C. Zhang, Science 314, 1757 (2006).

${ }^{4}$ M. Konig, S. Wiedmann, C. Brune, A. Roth, H. Buhmann, L. W. Molenkamp, X. Qi, and S. C. Zhang, Science 318, 766 (2007).

${ }^{5}$ H. Ohno, D. Chiba, and F. Matsukura, Nature (London) 408, 944 (2000).

${ }^{6}$ M. Fujita, K. Wakabayashi, K. Nakada, and K. Kusakabe, J. Phys. Soc. Jpn. 65, 1920 (1996).

${ }^{7}$ K. Wakabayashi, M. Sigrist, and M. Fujita, J. Phys. Soc. Jpn. 67, 2089 (1998).

${ }^{8}$ A. Yamashiro, Y. Shimoi, K. Harigaya, and K. Wakabayashi, Phys. Rev. B 68, 193410 (2003).

${ }^{9}$ Y. Son, M. L. Cohen, and S. G. Louie, Nature (London) 444, 347 (2006).

${ }^{10}$ Y.-W. Son, M. L. Cohen, and S. G. Louie, Phys. Rev. Lett. 97, 216803 (2006).

${ }^{11}$ E. Rudberg, P. Salek, and Yi Luo, Nano Lett. 7, 2211 (2007).

${ }^{12}$ O. Hod, V. Barone, J. Peralta, and G. E. Scuseria, Nano Lett. 7, 2295 (2007).

${ }^{13}$ K. S. Novoselov, A. K. Geim, S. V. Morozov, D. Jiang, M. I. Katsnelson, I. V. Grigorieva, S. V. Dubonos, and A. A. Firsov, Nature (London) 438, 197 (2005).

${ }^{14}$ Y. Zhang, Y. W. Tan, H. L. Stormer, and P. Kim, Nature (London) 438, 201 (2005).

${ }^{15}$ A. Geim and K. Novoselov, Nat. Mater. 6, 183 (2007).

${ }^{16}$ M. Y. Han, B. Ozyilmaz, Y. Zhang, and P. Kim, Phys. Rev. Lett. 98, 206805 (2007).

${ }^{17}$ B. Ozyilmaz, P. Jarillo-Herrero, D. Efetov, D. A. Abanin, L. S. Levitov, and P. Kim, Phys. Rev. Lett. 99, 166804 (2007).

${ }^{18}$ K. Nakada, M. Fujita, G. Dresselhaus, and M. S. Dresselhaus,
Phys. Rev. B 54, 17954 (1996).

${ }^{19}$ L. Brey and H. A. Fertig, Phys. Rev. B 73, 235411 (2006).

${ }^{20}$ F. Muñoz-Rojas, D. Jacob, J. Fernández-Rossier, and J. J. Palacios, Phys. Rev. B 74, 195417 (2006).

${ }^{21}$ J. Fernández-Rossier, J. J. Palacios, and L. Brey, Phys. Rev. B 75, 205441 (2007).

${ }^{22}$ J. W. Mintmire, J. Li, D. Gunlycke, and C. T. White, http:// meetings.aps.org/link/BAPS.2007.MAR.H28.12; M. Wimmer, I. Adagideli, S. Berber, D. Tomanek, and K. Richter, arXiv:0709.3244 (unpublished).

${ }^{23}$ J. Fernández-Rossier and J. J. Palacios, Phys. Rev. Lett. 99, 177204 (2007).

${ }^{24}$ Elliott H. Lieb, Phys. Rev. Lett. 62, 1201 (1989).

${ }^{25}$ D. Jérome, T. M. Rice, and W. Kohn, Phys. Rev. 158, 462 (1967).

${ }^{26}$ J. Fernández-Rossier and C. Tejedor, Phys. Rev. Lett. 78, 4809 (1997).

${ }^{27}$ J. Fernández-Rossier, C. Tejedor, and R. Merlin, Solid State Commun. 108, 473 (1998).

${ }^{28}$ Ji-Min Duan, D. P. Arovas, and L. J. Sham, Phys. Rev. Lett. 79, 2097 (1997).

${ }^{29}$ O. Hod, J. E. Peralta, and G. E. Scuseria, Phys. Rev. B 76, 233401 (2007).

${ }^{30}$ O. Hod, V. Barone, and G. E. Scuseria, Phys. Rev. B 77, 035411 (2008).

${ }^{31}$ Wei L. Wang, Sheng Meng, and E. Kaxiras, Nano Lett. 8, 241 (2008).

${ }^{32}$ Oleg V. Yazyev and M. I. Katsnelson, Phys. Rev. Lett. 100, 047209 (2008).

${ }^{33}$ F. Muñoz-Rojas, J. Fernández-Rossier, L. Brey, and J. J. Palacios, Phys. Rev. B 77, 045301 (2008).

${ }^{34}$ K. Sasaki, S. Murakami, and R. Saito, Appl. Phys. Lett. 88, 113110 (2006). 